\title{
Beim akuten Hörsturz Kortikoide einfach oral geben?
}

\author{
Gibt es Unterschiede in der Prognose, wenn Patienten mit akutem Hörsturz \\ systemisch oder intratympanal mit Kortikosteroiden behandelt werden?
}

$\mathrm{D}$ er Hörsturz tritt mit einer Inzidenz von 5-20 pro 100.000 Personen auf. Am häufigsten kommt dieses Krankheitsbild im Alter zwischen 45 und 55 Jahren vor. Etwa ein Drittel der Patienten weist zusätzlich vestibuläre Symptome auf. Ähnlich wie bei der Neuritis vestibularis wird hier eine Neuritis des achten Hirnnerven vermutet. Deshalb werden Patienten in der Regel mit Kortikosteroiden behandelt. Die hier vorliegende randomisierte Studie verglich die lokale mit der systemischen Steroidtherapie.

Es handelt sich um eine prospektive, randomisierte Nichtunterlegenheitsstudie von 250 Patienten mit einseitigem Hörsturz innerhalb von 14 Tagen nach Beginn der Symptome. Die Studie wurde an 16 Zentren in den USA durchgeführt. Die Patienten wurden über sechs Monate nachbeobachtet. Die Behandlung erfolgte entweder über 14 Tage mit $60 \mathrm{mg}$ oralem Prednison mit langsamem Ausschleichen oder mit vier konsekutiven Injektionen innerhalb von 14 Tagen mit 40 mg Methylprednisolon in das Mittelohr. Der primäre Endpunkt der Studie war die Änderung der Hörfunktion nach zwei Monaten. $121 \mathrm{~Pa}$ tienten wurden systemisch und 129 lokal behandelt. Das mittlere Alter betrug 51 Jahre; Männer waren häufiger betroffen als Frauen. Der Hörverlust war in Dezibel erheblich.

In der systemischen Therapiegruppe verbesserte sich das Hören um 30,7 Dezibel verglichen mit 28,7 Dezibel in der Injektionsgruppe. Das Hörvermögen zwei Monate nach der Behandlung betrug 56 versus 57,6 Dezibel. Bei Patienten mit akutem Hörsturz war demnach eine systemische Kortisonbehandlung genauso wirksam wie die Injektion von $\mathrm{Me}$ thylprednisolon in das Mittelohr.

Rauch S. D. et al. Oral vs intratympanic corticosteroid therapy for idiopathic sudden sensorineural hearing loss: a randomized trial. JAMA 2011305 2071-9
Kommentar: Es ist sehr wertvoll, dass jetzt für entzündliche Erkrankungen einzelner Hirnnerven in zunehmendem Maße prospektive, randomisierte Studien durchgeführt werden. So konnte die Überlegenheit einer Prednisontherapie sowohl für die periphere Facialisparese, die Neuritis vestibularis und den Hörsturz nachgewiesen werden. Die vorliegende Studie zeigt, dass die unkomplizierte orale Therapie mit Prednison, die auch viel preiswerter ist, genauso wirksam ist wie die aufwendige intratympanale Injektion von Methylprednisolon. Dies bedeutet, dass nach Ausschluss anderer Ursachen eines Hörsturzes die Behandlung problemlos im ambulanten Bereich durchgeführt werden kann.

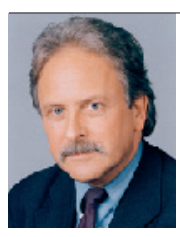

Prof. Dr. med.

Hans-Christoph Diener

Essen

\section{Fortgeschrittener Larynxkrebs: RT oder OP?}

Was die Radiochemotherapie von Plattenepithelkarzinomen des Kehlkopfes in fortgeschrittenen Tumorstadien leistet, haben italienische Forscher untersucht. Tatsächlich fallen die Ergebnisse gleich gut aus wie bei der Laryngektomie, solange der Primärtumor nicht das Stadium T4a erreicht hat.

$\mathrm{H}$ NO-Spezialisten und Radiologen der römischen Gemelli-Klinik sichteten für ihre Studie die Krankenakten von 65 konsekutiven Patienten, die zwischen November 2005 und Januar 2009 wegen eines fortgeschrittenen Plattenepithelkarzinoms des Larynx (Stadien III und IV) behandelt worden waren. Je nach Befund und Patientenpräferenz waren die Tumoren chirurgisch oder mit Chemoradiotherapie angegangen worden. Im letzteren Fall erhielten die Patienten neben der Bestrahlung noch Cisplatin bzw. Cetuximab.

Weder im Gesamtüberleben noch im krankheitsspezifischen Überleben unter- schieden sich beide Gruppen signifikant: $84 \%$ der Operierten und $64 \%$ der Bestrahlten lebten nach zwei Jahren noch $(\mathrm{p}=0,31)$, die krankheitsspezifische Überlebensrate betrug 84 vs. $80 \%$ (p = 0,95 ). Eine Ausnahme bildeten die klinisch als T4a eingestuften Tumoren: Hier überlebten nach totaler Laryngektomie $100 \%$ der Patienten zwei Jahre; in der Gruppe der Bestrahlten waren es nur $60 \%(\mathrm{p}=0,03)$.

$\mathrm{Ob}$ sich der Kehlkopf durch die Radiochemotherapie erhalten ließ, hing indessen stark vom Primärtumor ab. Die Erhaltungsraten betrugen $86 \%$ für
T2-Tumoren, $43 \%$ für T3- und $17 \%$ für T4a-Tumoren.

Fazit: Das Tumorstadium scheint zu entscheiden, ob eine Resektion oder eine Radiochemotherapie beim Larynxkarzinom sinnvoll ist. Patienten mit T4a-Tumoren benötigen eine totale Laryngektomie, bei Tumoren anderer Stadien zeigte sich hierfür kein Vorteil. Je weiter fortgeschritten der Tumor war, desto geringer war die Chance auf Erhaltung des Kehlkopfes durch eine Radiochemotherapie.

Dr. Robert Bublak

Bussu F et al. Oncologic outcomes in advanced laryngeal squamous cell carcinomas treated with different modalities in a single institution: a retrospective analysis of 65 cases. Head \& Neck 2011; doi: 10.1002/hed.21785. 